\title{
Juicios metacognitivos en la educación médica
}

\author{
Francisco Javier Ruiz Ortega a ; Jorge Norvey Álvarez Ríos ${ }^{\text {b }}$ \\ ${ }^{a} \mathrm{PhD}$ en Didáctica de las Ciencias Experimentales y las Matemáticas. Profesor, Universidad de Caldas.

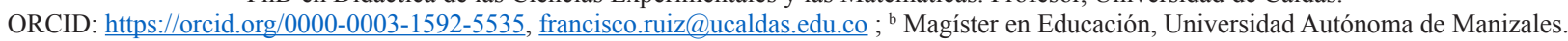 \\ ORCID: https://orcid.org/0000-0002-9110-9284, jnalvarez@autonoma.edu.co
}

DOI: https://doi.org/10.22517/25395203.24609

\section{Resumen}

En la educación médica, la metacognición incide en diferentes aspectos: mejora el rendimiento académico, contribuye a la toma de decisiones, facilita el desarrollo de habilidades clínicas, permite combatir el exceso de confianza y disminuye errores diagnósticos. Por ello, se constituye en una habilidad necesaria en la formación del médico. En este sentido, el uso de estrategias que promuevan el desarrollo de juicios metacognitivos, es clave en los procesos de enseñanza y aprendizaje. Para profundizar en los aportes de los juicios metacognitivos en el campo de la educación médica, se construye el siguiente artículo producto de una revisión bibliográfica. En él se logra pasar de una dimensión macro de la metacognición (conocimiento o regulación), a una dimensión micro referida a los juicios metacognitivos, evidenciado cómo estos aportan al razonamiento clínico, a la toma de decisiones, a la disminución del error médico y a la seguridad del paciente. El artículo muestra una línea de trabajo denominada juicios metacognitivos, caracterizada por centrarse en la precisión entre las afirmaciones de desempeño en una tarea y el rendimiento real.

Palabras claves: Juicios metacognitivos, Metacognición, Aprendizaje, Educación médica (Decs)

\section{Metacognitive judgments in medical education}

\begin{abstract}
Metacognition contributes to medical education from different aspects: improve academic performance, contributes to decision making, facilitates the development of clinical skills, helps combat overconfidence and reduces diagnostic errors. This is why it becomes a necessary skill to develop in the training of the student medicine for which strategies such as metacognitive scaffolds are included, deliberate consideration of alternatives, and the reproduction of optimal actions. To understand these contributions for learning medicine, a bibliographic review article was developed, through a process that included searching databases through descriptors in Spanish and English, the selection of articles that will include the relationship of metacognition and the learning of medicine, to arrive at the construction of a text that would account for an understanding of the subject. The above allowed, not just delve into metacognition as a higher order skill in learning, but also guide a discussion that highlights a line of work called metacognitive judgments, which is characterized by focusing on the precision between performance claims on a task and actual performance.

Keywords: Metacognition, Metacognitive judments, Learning, Medical education, (MESH)
\end{abstract}

\section{Introducción}

El aprendizaje de la medicina implica el análisis de elementos como la adquisición, transferencia y retención del conocimiento (1-3). De igual manera, la comprensión de elementos más profundos como el desarrollo de la capacidad individual de autoorganización y la acción. Esto, sin duda, facilita que el estudiante, además de realizar ajustes al abordar situaciones desconocidas y comprender sus límites y sesgos (4), reconozca que cada día los problemas son más desafiantes y que por lo tanto, exigen acciones efectivas y tomar decisiones pertinentes para solucionarlos. En definitiva, se requiere la activación de habilidades y recursos personales indispensables en el análisis de una situación, la reflexión activa de las competencias y el conocimiento de cómo se construye el conocimiento, también conocida como metacognición (4).

La metacognición es una de las habilidades de orden superior, definida como el conocimiento de los propios pensamientos y los diferentes factores que pueden influir sobre ellos (5). En el campo del aprendizaje se le reconoce por sus aportes 
en la conciencia del proceso de estudio, el monitoreo de la eficacia en el desarrollo de la tarea y la adaptación a las exigencias de la actividad (6). Desde el campo clínico, es una estrategia que pueden utilizar los médicos para reflexionar sobre el proceso de pensamiento en el contexto inmediato de la decisión clínica (7). Es decir, aporta al proceso de razonamiento clínico y a la toma de decisiones.

Ahora, en el campo de la educación médica, la metacognición se incorpora a través de diferentes formas, entre ellas: el monitoreo en la toma de decisiones y disminución de sesgos retrospectivos (8); como un método para para promover el almacenamiento, recuperación, transferencia y aplicación de las ciencias básicas (9); finalmente, como una estrategia para mejorar la toma de decisiones que conduzcan al éxito en el desarrollo de la tarea (10). En la actualidad, las nuevas líneas de investigación y teorías en el campo de la metacognición ubican sus conceptualizaciones y propuestas metodológicas en los denominados juicios metacognitivos, referidos a estimaciones sobre aciertos $\mathrm{y}$ desaciertos de acuerdo a una tarea y un objetivo propuesto (11). La literatura revisada muestra que los juicios pueden clasificarse como juicios de dificultad, juicios de aprendizaje, juicios de confianza, juicios de sentimientos de saber y juicios de rendimiento (11).

En este sentido, el presente artículo pretende, a través de una revisión sistemática, ubicar teóricamente los avances de la metacognición. Lo anterior con un especial énfasis en la línea de investigación de los juicios metacognitivos aplicados al aprendizaje de la medicina, a través de un proceso de consulta de artículos científicos ubicados en diferentes bases de datos y un análisis de los aportes de los autores.

\section{Metodología}

Se utilizaron para la búsqueda de los documentos las bases de datos: Web of Science, Science Direct, Scopus . Se determinaron como buscadores: Eric y Pubmed. Los descriptores en inglés y español, respectivamente, fueron los siguientes: metacognition, learning, metacognition judgment, metacognición, aprendizaje y medicina. El resultado de este proceso arrojó un total de 532 artículos. Los criterios de selección fueron:1) Publicados en los últimos 5 años; 2) Artículos que expongan una relación explícita entre metacognición y el aprendizaje de la medicina. A partir de estos criterios, se formó un banco de datos con 37 documentos (Tabla 1).

\begin{tabular}{lll}
\hline Base de datos & $\begin{array}{l}\text { Número de } \\
\text { artículos iden- } \\
\text { tificados }\end{array}$ & $\begin{array}{l}\text { Número de artículos } \\
\text { seleccionados }\end{array}$ \\
\hline Web of Science & 38 & 12 \\
Scopus & 66 & 10 \\
Science Direct & 237 & 1 \\
PubMed & 114 & 7 \\
Eric & 77 & 7 \\
Total & 532 & 37 \\
\hline \multicolumn{3}{c}{ Tabla 1: Artículos de investigación } \\
sobre metacognición.
\end{tabular}

\section{Discusión}

Inicialmente, se describen los aportes de la metacognición al aprendizaje de la medicina. De manera posterior, se profundizará en los juicios metacognitivos; línea de investigación emergente que trabaja sobre la precisión en las afirmaciones de aciertos y desaciertos con relación al desempeño en una tarea.

\section{Metacognición}

Para algunos autores, la metacognición se asocia con mejores resultados en el rendimiento académico, el éxito clínico y el aprendizaje permanente (12-15). Sin embargo, en el estudio desarrollado por Baothman et al (6), se demuestra que la metacognición y la autorregulación no se correlacionan con mejores resultados en las pruebas. Esta diferencia entre autores depende, significativamente, del tipo de variables medidas y el tipo de pruebas estandarizadas que miden el desempeño del estudiante durante el desarrollo de una tarea.

El rastreo bibliográfico muestra una tendencia en el desarrollo de investigaciones que buscan correlacionar los diferentes componentes de la metacognición (conocimiento metacognitivoy regulaciónmetacognitiva) con el desempeño en pruebas estandarizadas. En este sentido, el estudio de Khalil et al (13), correlacionan el inventario de estrategias de aprendizaje con el éxito académico; estudio que demuestra una relación entre la autorregulación (gestión del tiempo, autoevaluación y ayuda al estudio) y el rendimiento académico del estudiante. Asimismo, Lafleur et al (16) trabajan sobre la correlación entre la metacognición y los exámenes clínicos objetivos estructurados (ECOE); este estudio pone en evidencia la modificación en la gestión del tiempo para el desarrollo de este tipo de evaluaciones.

Con las diferentes pruebas, relaciones y análisis, se demuestra que la metacognición aporta al razonamiento clínico y a la toma de decisiones (17). De manera especial, permite trabajar sobre los sesgos cognitivos, 
definidos estos como desviaciones de la racionalidad, ya que, la metacognición facilita la autorreflexión crítica por medio de la separación del contexto inmediato en el momento que se toma de la decisión con el propósito de analizar el proceso de pensamiento utilizado $(7,17,18)$.

Las investigaciones, en este campo, ponen en evidencia que los estudiantes de medicina cuentan con dificultades importantes para aplicar el conocimiento conceptual a los casos clínicos y que carecen de conciencia metacognitiva (17). Esto implica una invitación a que en el aula de clase de medicina se trabaje la metacognición con el fin de lograr que los estudiantes desarrollen la capacidad de realizar una preparación previa al desarrollo de la tarea, ajustar sus comportamientos de acuerdo al rendimiento durante la tarea y realizar una autorreflexión a través de la evaluación de sus propios resultados (15). A partir de los autores anteriores, se puede evidenciar que existe la necesidad del abordaje de la metacognición en la formación del médico; además de asumir que ésta se constituye en una habilidad que favorece el aprendizaje del razonamiento clínico, la toma de decisiones y el análisis de las mejores alternativas.

\section{Juicios metacognitivos}

La revisión bibliográfica reafirma el surgimiento de nuevas líneas de investigación, entre ellas los juicios metacognitivos (JM), asumidos como aquellas afirmaciones sobre los aciertos y desaciertos en el desarrollo de una tarea. Los JM, pueden darse según el momento en que se evalué (11) el desarrollo de una actividad. En este sentido, podemos encontrar juicios de dificultad, de aprendizaje, de confianza y de rendimiento; todos ellos, posibles de evaluarse antes (juicios prospectivos), durante (juicios concurrentes) y después del desarrollo de la tarea. (juicios retrospectivos) $(11,21$, 22)

En cuanto a la calibración, surgen de un lado medidas de precisión absoluta que es coincidencia exacta entre el juicio emitido y el rendimiento y la de precisión relativa que evidencia los juicios de confianza correctos o incorrectos (20). De otro lado, se tienen medidas como: sensibilidad y especificidad (21). La primera, entendida como la capacidad de discriminar entre respuestas correctas e incorrectas (22) y se calcula como la proporción de juicios afirmativos cuando el ítem se responde correctamente; con ella además, se puede medir el sentimiento de conocimiento, la precisión de rendimiento y la facilidad de procesamiento (20). La segunda medida denominada de especificidad, indica la proporción de juicios negativos, cuando el ítem se responde correctamente (21); por medio de esta medida se puede calcular el sentimiento de no saber y la dificultad de procesamiento (20).

Los anteriores elementos posibilitan el desarrollo de diferentes investigaciones que ponen en evidencia que los juicios metacognitivos son: a) predictores de mejores rendimientos, reafirmando a su vez, b) dependen de los conocimientos previos (entre mayor conocimiento previo se tenga de la tarea es mayor la precisión), c) aportan a la resolución de problemas (23). Asimismo, que la precisión de los juicios metacognitivos en los primeros años de medicina se da mejor en tareas más pequeñas (24). Ahora bien, desde la formación de los futuros profesionales en el campo de la salud, se identifica que contribuyen a la disminución del exceso de confianza respecto a la tarea, mejorar el proceso diagnóstico y mejorar la toma de decisiones; todos indispensables para la seguridad del paciente $(24,25)$; el estudiante, al realizar un análisis de proceso de aprendizaje, identifica a la vez qué signos y síntomas ha pasado por alto en el proceso de valoración, además de que otras valoraciones se requieren para tomar una mejor decisión. Motivos relevantes que indican la necesaria incorporación de los juicios metacognitivos en la formación del médico.

\section{Juicios de confianza}

Los juicios de confianza se definen como la estimación de una persona que toma una decisión sobre la posibilidad de acertar (26). Corresponden a uno de los juicios metacognitivos que se miden en el antes, el durante y el después de una tarea. De acuerdo a la revisión realizada, es uno de los juicios más evaluados en los estudiantes de medicina e incluso en el campo clínico, quizás, por su relación con que el exceso de confianza es uno de los factores que podría incidir con mayor peso a que se presente el error médico en el diagnóstico (27). Las investigaciones reportadas evidencian una tendencia a la sobreestimación del desempeño real por parte de los estudiantes. En este sentido, Callender et al (28) y Fritzsche et al (29), identifican que los estudiantes de medicina que tienen menor desempeño en la tarea tienen un exceso de confianza. Asimismo, De Bruin et al (30) muestran que los estudiantes universitarios tienen un exceso de confianza en los resultados de los exámenes; situación que conlleva a problemas de autorregulación y el desempeño académico.

Los trabajos reportados muestran que la precisión de los juicios metacognitivos y la disminución del exceso de confianza se puede mejorar a través de procesos de formación en juicios metacognitivos (31); y, para ello, el andamiaje metacognitivo puede ser una de las estrategias más relevantes en el campo de la enseñanza 
de la metacognición e incorporación de los juicios en el aula de clases.

Al describir el andamiaje metacognitivo (articulado a la teoría vigotskiana (35)), se hace referencia a la metáfora de andamio, en el cual se brinda un apoyo temporal al estudiante en tareas complejas, que no hubiesen tenido éxito en su ejecución, sin la guía del docente. En el campo de la medicina, se da de manera particular cuando el estudiante empieza a tomar decisiones producto del razonamiento clínico. Al aplicar esta estrategia, se debe tener en cuenta que es un proceso secuencial que aumenta la complejidad de manera gradual que pasa de habilidades globales a habilidades locales (8).

\section{Juicios de aprendizaje y de sentimientos de saber}

Finalmente, están los juicios de aprendizaje y los juicios de sentimientos de saber. En este caso se logró identificar dos investigaciones. Primero, el trabajo de Avhusttiuk et al (32) quienes abordan el juicio de sentimiento de saber en estudiantes universitarios; su estudio demuestra tres aspectos importantes: primero, que el juicio se da con mayor frecuencia en los juicios prospectivos; segundo, que dependen del tipo de tarea y tercero, que se presentan en estudiantes más jóvenes con rendimientos académicos más bajos. Por otro lado, se logró identificar el estudio de Andreas et al (33), los autores, al evaluar la precisión de los juicios de aprendizaje, ponen en evidencia que trabajarlos en el aula de clase facilita su precisión.

\section{Conclusiones}

Después de la revisión de los anteriores artículos se pueden llegar a las siguientes conclusiones:

1) Se debe dar paso al desarrollo teórico y metodológico de la metacognición, sustentado en la comprensión de elementos micro, es decir, a la incorporación de estudios sobre cómo los juicios de diferente naturaleza metacognitiva, contribuyen al mejoramiento de la precisión del desempeño de los estudiantes frente a una tarea específico al enriquecer los procesos de razonamiento clínico y con esto la toma de decisiones.

2) Al incorporar en el campo clínico los procesos de valoración, de razonamiento clínico y de toma de decisiones de una manera más detallada sobre una tarea, se conduce a la disminución de sesgos y de errores médicos.

3) Los juicios de confianza, los más estudiados en medicina, demuestran que existe, con frecuencia, un exceso de confianza que puede llevar a un bajo desempeño académico. Trabajarlos, en el aula de clase, seguramente posibilitan un aprendizaje más consciente y analítico, aspectos indispensables en la apropiación del conocimiento y de los procesos que debe realizar el profesional de este campo y necesarios, además, para reducir la brecha entre los juicios emitidos y el rendimiento real.

Se recomienda, entonces, que las programaciones del docente proyectadas al desarrollo de interacciones comunicativas con sus estudiantes, estén permeadas de escenarios de orden metacognitivo, en las cuales, la reflexión sobre sus propios juicios y procesos, sea una actividad explícita, consciente e intencionada, para favorecer el desarrollo de aprendizajes profundos en los estudiantes. Incorporar los juicios metacognitivos en el aula, seguramente permitirá al docente sustentar los procesos de enseñanza más que en contenidos, en procesos de pensamiento de orden superior, y a los estudiantes, pasar de un aprendizaje superficial a un aprendizaje en profundidad.

\section{Referencias}

1. Zare S, Hemmatjo R, Allahyari T, Hajaghazadeh M, Hajivandi A, Aghabeigi M, et al. Comparison of the effect of typical firefighting activities, live fire drills and rescue operations at height on firefighters' physiological responses and cognitive function. Ergonomics. 2018;61(10):1334-44.

2. Arcoraci V, Squadrito F, Altavilla D, Bitto A, Minutoli L, Penna O, et al. Medical simulation in pharmacology learning and retention: A comparison study with traditional teaching in undergraduate medical students. Pharmacol Res Perspect [Internet]2019 [cited Jun, 2019];7(1). Available from: https://www.ncbi.nlm.nih.gov/ pmc/articles/PMC6327107/

3. Alluri RK, Tsing P, Lee E, Napolitano J. A randomized controlled trial of high-fidelity simulation versus lecture-based education in preclinical medical students. Med Teach. 2016;38(4):404-9.

4. Hasske E, Beil M, Keller K. Competencybased Education and Training of medical staff. A Programm of the Medical Academy Waldbreitbach: Concept - Implementation Materials. GMS J Med Educ [Internet]2017 [cited Apr 27,2020];34(4). Available from: https://www. ncbi.nlm.nih.gov/pmc/articles/PMC5654121/ 
5. Poorman SG, Mastorovich ML. Using Metacognitive Wrappers to Help Students Enhance Their Prioritization and Test-Taking Skills. Nurse Educ. 2016;41(6):282-5.

6. Baothman A, AlJefri H, Agha S, Khan MA. Study Habits of Health Science Students at King Saud bin Abdulaziz University for Health Sciences, Jeddah, Saudi Arabia. SAGE Open. 2018;8(2):2158244018778092.

7. Chew KS, Durning SJ, vanMerriënboerJJ.Teaching metacognition in clinical decision-making using a novel mnemonic checklist: an exploratory study. Singapore. Med J. 2016;57(12):694-700.

8. Medina MS, Castleberry AN, Persky AM. Strategies for Improving Learner Metacognition in Health Professional Education. Am J Pharm Educ [Internet]. mayo de 2017 [cited Aug 18, 2019];81(4). Available from: https://www.ncbi. nlm.nih.gov/pmc/articles/PMC5468716/

9. Hennrikus EF, Skolka MP, Hennrikus N. Applying Metacognition Through Patient Encounters and Illness Scripts to Create a Conceptual Framework for Basic Science Integration, Storage, and Retrieval. J Med Educ Curric Dev [Internet] 2018 [cited Dec16,2020];5. Available from: https://www.ncbi.nlm.nih.gov/pmc/articles/ PMC5967154/

10. Ali S, Yasmeen R. Practice to preach selfregulation: Use of metacognitive strategies by medical teachers in their learning practices. Pak J Med Sci. 2019;35(6):1642-6.

11. Valenzuela A. ¿Qué hay de nuevo en la metacognición? Revisión del concepto, sus componentes y términos afines. Educ Pesqui Sao Paulo. 2019;15(187571).

12. Hong WH, Vadivelu J, Daniel EGS, Sim JH. Thinking about thinking: changes in firstyear medical students' metacognition and its relation to performance. Med Educ Online [Internet]2015 [cited Dec 14,2020];20. Available from: https://www.ncbi.nlm.nih.gov/pmc/articles/ $\underline{\mathrm{PMC} 4551498 /}$

13. Khalil MK, Williams SE, Gregory Hawkins H. Learning and study strategies correlate with medical students' performance in anatomical sciences. Anat Sci Educ. 2018;11(3):236-42.

14. Cho KK, Marjadi B, Langendyk V, Hu W. Medical student changes in self-regulated learning during the transition to the clinical environment. BMC Med Educ. 2017;17(1):59.

15. Siqueira MAM, Gonçalves JP, Mendonça VS, Kobayasi R, Arantes-Costa FM, Tempski PZ, et al. Relationship between metacognitive awareness and motivation to learn in medical students. BMC Med Educ [Internet] 2020 [cited Dec 15,2020];20. Available from https://www.ncbi.nlm.nih.gov/ pmc/articles/PMC7602298/

16. Lafleur A, Laflamme J, Leppink J, Côté L. Task Demands in OSCEs Influence Learning Strategies. Teach Learn Med. 2017;29(3):286-95.

17. Kiesewetter J, Ebersbach R, Tsalas N, Holzer M, Schmidmaier R, Fischer MR. Knowledge is not enough to solve the problems - The role of diagnostic knowledge in clinical reasoning activities. BMC Med Educ. 2016;16(1):303.

18. Chew KS, van Merrienboer JJG, Durning SJ. Perception of the usability and implementation of a metacognitive mnemonic to check cognitive errors in clinical setting. BMC Med Educ [Internet] 2019 [cited Feb 10,2019];19. Available from: https://www.ncbi.nlm.nih.gov/pmc/articles/ PMC6327396/

19. Craig K, Hale D, Grainger C, Stewart ME. Evaluating metacognitive self-reports: systematic reviews of the value of self-report in metacognitive research. Metacognition Learn. 2020;15(2):155213.

20. Gutiérrez AP, Schraw G, Kuch F, Richmond AS. A two-process model of metacognitive monitoring: Evidence for general accuracy and error factors. Learn Instr. 2016;44:1-10.

21. Händel $\mathrm{M}$, de Bruin $\mathrm{ABH}$, Dresel M. Individual differences in local and global metacognitive judgments. Metacognition Learn. 2020.

22. Mazancieux A, Dinze C, Souchay C, Moulin CJA. Metacognitive domain specificity in feelingof-knowing but not retrospective confidence. Neurosci Conscious [Internet]. 24 de febrero de 2020 [cited Dec 14, 2020];2020(1). Available from: https://www.ncbi.nlm.nih.gov/pmc/articles/ PMC7043299/

23. Mihalca L, Mengelkamp C, Schnotz W. Accuracy of metacognitive judgments as a moderator of learner control effectiveness in problem-solving 
tasks. Metacognition Learn. 1 2017;12(3):357-79.

24. Cleary TJ, Konopasky A, La Rochelle JS, Neubauer BE, Durning SJ, Artino AR. First-year medical students' calibration bias and accuracy across clinical reasoning activities. Adv Health Sci Educ. 2019;24(4):767-81.

25. Garcia-Retamero R, Cokely E, Hoffrage U. Visual Aids Improve Diagnostic Inferences and Metacognitive Judgment Calibration. Front Psychol. 2015;6:932.

26. Lebreton M, Bacily K, Palminteri S, Engelmann JB. Contextual influence on confidence judgments in human reinforcement learning. PLoS Comput Biol [Internet]. 8 de abril de 2019 [cited Dec15, 2020];15(4). Available from: https:/www.ncbi. nlm.nih.gov/pmc/articles/PMC6472836/

27. Braun LT, Zwaan L, Kiesewetter J, Fischer MR, Schmidmaier R. Diagnostic errors by medical students: results of a prospective qualitative study. BMC Med Educ [Internet] 2017 [cited Feb 13, 2019];17. Available from: https://www.ncbi.nlm. nih.gov/pmc/articles/PMC5679151/

28. Callender AA, Franco-Watkins AM, Roberts AS. Improving metacognition in the classroom through instruction, training, and feedback. Metacognition Learn. 2016;11(2):215-35.

29. Fritzsche ES, Händel M, Kröner S. What do second-order judgments tell us about lowperforming students' metacognitive awareness? Metacognition Learn. 2018;13(2):159-77.

30. de Bruin ABH, Kok EM, Lobbestael J, de Grip A. The impact of an online tool for monitoring and regulating learning at university: overconfidence, learning strategy, and personality. Metacognition Learn. 2017;12(1):21-43.

31. Melgar MF, Elisondo R, Melgar MF, Elisondo R. Metacognicióny buenas prácticas en la universidad. ¿Qué aspectos valoran los estudiantes? Innov Educ México DF. 2017;17(74):17-38.

32. Avhustiuk MM, Pasichnyk ID, Kalamazh RV. The Illusion of Knowing in Metacognitive Monitoring: Effects of the Type of Information and of Personal, Cognitive, Metacognitive, and Individual Psychological Characteristics. Eur J Psychol.2018;14(2):317-41.

33. Andreas J, Veit K, Fredik J. What moderates the accuracy of ease of learning judgments? Metacognition Learn. 2017; 12 (3) 1 - 19 